\title{
The Radio Spectrum of TVLM513-46546: Constraints on the Coronal Properties of a Late M Dwarf
}

\author{
Rachel A. Osten ${ }^{1}$ \\ National Radio Astronomy Observatory, 520 Edgemont Road, Charlottesville, VA 22903; \\ rosten@nrao.edu \\ Suzanne L. Hawley \\ Astronomy Department, Box 351580, University of Washington, Seattle WA 98195; \\ slh@astro.washington.edu \\ Timothy S. Bastian \\ NRAO, Charlottesville,VA 22903; tbastian@nrao.edu \\ I. Neill Reid \\ Space Telescope Science Institue, 3700 San Martin Drive, Baltimore MD 21218; \\ inr@stsci.edu
}

\begin{abstract}
We explore the radio emission from the M9 dwarf, TVLM513-46546, at multiple radio frequencies, determining the flux spectrum of persistent radio emission, as well as constraining the levels of circular polarization. Detections at both 3.6 and $6 \mathrm{~cm}$ provide spectral index measurement $\alpha$ (where $\mathrm{S}_{\nu} \propto \nu^{\alpha}$ ) of $-0.4 \pm 0.1$. A detection at $20 \mathrm{~cm}$ suggests that the spectral peak is between 1.4 and $5 \mathrm{GHz}$. The most stringent upper limits on circular polarization are at 3.6 and $6 \mathrm{~cm}$, with $V / I<15 \%$. These characteristics agree well with those of typical parameters for early to mid $\mathrm{M}$ dwarfs, confirming that magnetic activity is present at levels comparable with those extrapolated from earlier M dwarfs. We apply analytic models to investigate the coronal properties under simple assumptions of dipole magnetic field geometry and radially varying nonthermal electron density distributions. Requiring the spectrum to be optically thin at frequencies higher than $5 \mathrm{GHz}$ and reproducing the observed $3.6 \mathrm{~cm}$ fluxes constrains the magnetic field at the base to be less than about $500 \mathrm{G}$. There is no statistically significant periodicity in the $3.6 \mathrm{~cm}$ light curve, but it is consistent with low-level variability.
\end{abstract}

\footnotetext{
${ }^{1}$ Jansky Postdoctoral Fellow
} 
Subject headings: stars: activity, stars: coronae, stars: late-type, radio continuum

\section{Introduction}

The detection of magnetic activity signatures in very late-type dwarfs (late $\mathrm{M} \rightarrow \mathrm{L}$ ) is puzzling, as the fraction of objects showing $\mathrm{H} \alpha$ in emission, the traditional activity signature, declines precipitously past type M8 (West et al. 2004). The rotation-activity correlation found in earlier type stars ( $\mathrm{F}$ to early $\mathrm{M}$ ) appears to break down: most objects M9 and cooler have rapid rotation, but weak or undetectable $\mathrm{H} \alpha$ emission (Mohanty \& Basri 2003). Models suggest (Mohanty et al. 2002) that the highly neutral atmospheres of these ultracool objects should be incapable of sustaining the kinds of magnetic stresses which pervade the atmospheres of solar-like stars ( $\mathrm{G}, \mathrm{K}$, early $\mathrm{M}$ ) and participating in the non-radiative heating of chromospheric and coronal plasma. And yet, transition region, X-ray, and radio persistent emission is clearly detected in some objects (Hawley \& Johns-Krull 2003; Fleming et al. 2003; Berger 2002), along with flare-like variability (Rutledge et al. 2000; Liebert et al. 2003). Transition region and X-ray observations detect persistent fluxes at levels compatible with scaling laws (based on $\mathrm{H} \alpha$ ) extrapolated from earlier type $\mathrm{M}$ dwarfs. The detection of both persistent and flaring radio emission from a handful of late $\mathrm{M}$ and L objects, reported in Berger et al. (2001) and Berger (2002), suggests that nonthermal radio emission is also present at these late spectral types. The interpretation of the radio emission, however, is complicated by the fact that many potential mechanisms may be at work. In an effort to better understand the radio emission from late $\mathrm{M}$ dwarfs, we undertook a deep pointing of an object previously detected at a single radio frequency.

TVLM513-46546 (=2MASS J15010818+2250020) is a young disk M9 dwarf, located at a distance of $10.5 \mathrm{pc}$. It was first detected in Tinney et al. (1993). It has $\mathrm{H} \alpha$ emission which is variable (equivalent width measurements of $1.8 \AA$ and $3.5 \AA$; Mohanty \& Basri 2003; Reid et al. 2002), and rapid rotation (vsin $i \sim 60 \mathrm{~km} \mathrm{~s}^{-1}$ ) corresponding to a minimum rotation period of 2.4 hours (see $\S 5$ ). There is no evidence of a physical companion between 0.1 " and 5" (Close et al. 2003). Luminosity and effective temperature estimates from Leggett et al. $(2001)$ are $\log \left(L_{\mathrm{bol}} / L_{\odot}\right) \sim-3.65$ and $\mathrm{T}_{\text {eff }} \sim 2200 \mathrm{~K}$. The lack of lithium in the spectrum constrains it to be more massive than $0.06 \mathrm{M}_{\odot}$ and thus likely a very low mass star rather than a brown dwarf. TVLM513-46546 (hereafter, TVLM513) was detected in the radio at a wavelength of $3.6 \mathrm{~cm}$ by Berger (2002), who gave an average flux density of $308 \pm 16 \mu \mathrm{Jy}$, with evidence of variability (peak flux of $1100 \mu \mathrm{Jy}$ ). 


\section{Data Reduction}

Observations were made on 2004 January 24 with the NRAO Very Large Array (VLA) ${ }^{1}$ when the array was being reconfigured from $\mathrm{B}$ to $\mathrm{C}$ configuration. The observations spanned $\sim 11.3 \mathrm{hrs}$, using receivers at 20,6, and $3.6 \mathrm{~cm}$. The flux density calibrator was 3C286, with assumed flux densities at 20,6, and $3.6 \mathrm{~cm}$ of $14.5,7.4$, and $5.2 \mathrm{Jy}$, respectively. The phase calibrator at all frequencies was $1513+236$, with flux densities of $1.7,0.8$, and $0.5 \mathrm{Jy}$ at 20,6 and $3.6 \mathrm{~cm}$, respectively. Data reduction was performed in AIPS. The observing strategy involved time sharing between frequency bands, with approximately 10 minutes on source per frequency; we reduced each frequency separately, first imaging the field of view to determine if the object was detected, then removing all background sources from the visibility dataset and re-imaging the field. A two-dimensional Gaussian was fit to the source, deriving intensity and source location. For frequencies at which a positive detection was made, the discrete Fourier transform of the visibilities was made as a function of time using the AIPS task DFTPL to examine variability. Images in Stokes V were also made to search for any evidence of circularly polarized flux.

\section{Analysis}

\subsection{Flux and Polarization Spectra}

TVLM513 was strongly detected at 6 and $3.6 \mathrm{~cm}$, with a marginal detection $(5.6 \sigma)$ at $20 \mathrm{~cm}$. Table 1 describes the parameters of the radio source at each frequency for the timeintegrated data. The derived spectral index between 6 and $3.6 \mathrm{~cm}$ is $-0.4 \pm 0.1$, and between 20 and $6 \mathrm{~cm}$ it is $0.1 \pm 0.2$. The upper limits $(3 \sigma)$ on the amount of circularly polarized flux at 3.6 and $6 \mathrm{~cm}$ lead to constraints on the circular polarization $\mathrm{V} / \mathrm{I}$ of $<15 \%$. The persistent nature of the radio flux as well as the lack of circular polarization signal points to a uniform coverage of the stellar disk with magnetic fields, either large or small-scale.

We computed the distribution of 6-3.6 cm spectral indices from a small sample of radio observations of dMe and dKe stars, compiled by Güdel \& Benz (1996). Although there is a fair amount of scatter (the slope errors range from 0.05-0.5), the distribution can be fit well by a Gaussian with FWHM=1.5, centered at -0.41 , which agrees with the measured value for TVLM513. The upper limits on circular polarization are also consistent with the general trends of quiescent radio emission from dMe stars (White et al. 1989).

\footnotetext{
${ }^{1}$ The NRAO is a facility of the National Science Foundation operated under cooperative agreement by Associated Universities, Inc
} 
Estimation of the brightness temperature requires separating the observed source flux density from the source size. We can place conservative limits by assuming the source extent to be the stellar diameter; at $10.5 \mathrm{pc}$, the fluxes listed in Table 1 imply brightness temperatures of a few times $10^{9} \mathrm{~K}$. This is consistent with gyrosynchrotron radiation.

\subsection{Variability}

Figure 1 plots the variation of TVLM513 at the two frequencies in which it was strongly detected during the course of the observation. During the observations of TVLM513 reported in Berger (2002) a flare (factor of 4 increase in flux density) over $\approx 25$ minutes was recorded. That radio flare was accompanied by large values of circular polarization (reaching $\sim 70 \%$ ). In contrast, our observations appear to be characterized by quiescent conditions.

A Lomb normalized periodogram of the $3.6 \mathrm{~cm}$ light curve reveals peaks at frequencies corresponding to periods 2.1 and $2.8 \mathrm{hr}$, although the false-alarm probability is significant ( 0.22 and 0.54 for 2.1 and $2.8 \mathrm{hr}$ periods, respectively). There are no strong periods in the $6 \mathrm{~cm}$ light curve. We examined the periods in the $3.6 \mathrm{~cm}$ light curve by imaging the target in each $\approx 10$ minute scan, and fitting a two-dimensional Gaussian to the point source. The variation of the peak intensity found this way matches those determined from the DFTPL program, and the same periodicities are produced from a power spectrum of the light curve. We would expect a minimum rotation period for TVLM513 of 2.4 hours, given the $v \sin i$ of $60 \mathrm{~km} \mathrm{~s}^{-1}$ and a stellar radius of $0.12 \mathrm{R}_{\odot}$ (based on direct mass and radius determination for

OGLE-TR-122b of $\mathrm{M}=0.092 \pm 0.009 \mathrm{M}_{\odot}, \mathrm{R}=0.120_{-0.013}^{+0.024} \mathrm{R}_{\odot}$ by radial velocity variations and transit depths, Pont et al. 2005).

We performed $\chi^{2}$ tests to determine whether the data were consistent with no variability, by comparing the fluxes with the average flux density from the observation. At $3.6 \mathrm{~cm}$, the average flux is $239 \pm 73 \mu \mathrm{Jy}$, with $\chi^{2}$ fit to the average returning $\chi_{\nu}^{2}=2.3$ (17 dof), indicating agreement at the $0.2 \%$ level for a constant source. At $6 \mathrm{~cm}$, the average flux is $278 \pm 63 \mu \mathrm{Jy}$, and a $\chi^{2}$ fit to the average yields $\chi_{\nu}^{2}=1.2$ (17 dof), indicating agreement at the $25 \%$ level for a constant source. We note that the time sampling, 10 minutes in each frequency band every 36 minutes, is relatively coarse, and this together with the low amplitude of the variation may affect the statistical significance. Thus we can conclude that there is marginal evidence for variations of the signal from TVLM513 at $3.6 \mathrm{~cm}$, but not $6 \mathrm{~cm}$.

Previous observations of TVLM513 with the VLA lasted 6200 seconds (23 September 2001, reported in Berger 2002) and 1350 seconds, respectively (unpublished observations of 15 February 1994). Neither had a sufficient time baseline to identify variability on timescales $\geq$ 
2 hours; it is unlikely that any periodicities could be seen in those data. Adding the current observations to this time baseline reduces the flare duty cycle reported in Berger (2002), based on one flare lasting $\sim 1500$ s, to $\sim 3 \%$.

\section{Coronal Models}

The brightness temperatures inferred in $\S 3.1$ are too large for thermal bremsstrahlung emission to be the cause. Particle acceleration, leading to gyrosynchrotron emission, is the more probable source. The spectral shape implies that frequencies greater than $5 \mathrm{GHz}$ are optically thin, and the observed $5-8 \mathrm{GHz}$ spectral index is compatible with optically thin emission from a relatively hard energy distribution with power law index $\delta \sim 2$ or 3 . The radio characteristics of TVLM513 are very similar to those of active dMe stars, in terms of spectral index and constraints on polarization levels. We investigate simple models for the coronal properties, under the assumption that the emission mechanism is gyrosynchrotron radiation from a population of mildly relativistic electrons: first, for a homogeneous source, and second, for a dipole magnetic field and radially varying nonthermal electron density. These two approaches give different, but consistent, results for the magnetic field strengths in the TVLM513's corona and the total number density of accelerated particles.

The turnover in spectral index between 1.4 and $4.8 \mathrm{GHz}$ suggests that the spectral maximum is in this range. The spectral turnover could be due to a transition between optically thick/thin regimes; if it is, the peak frequency of gyrosynchrotron emission depends on the magnetic field strength in the radio-emitting source, the source size, and index of the powerlaw distribution of nonthermal electrons. The strongest dependence of these parameters is from the magnetic field strength. For the simplest case of a homogeneous radio-emitting source, we constrain the magnetic field strength using equation (39) of Dulk (1985), setting $\delta=2(3)$, source size $\sim R_{\star}-2 R_{\star}$, and the total number density of nonthermal electrons above a cutoff value of $10 \mathrm{keV}$ between $10^{5}-10^{6} \mathrm{~cm}^{-3}$. Field strengths of a few hundred Gauss are needed in a homogeneous radio-emitting source to produce a spectral turnover below $5 \mathrm{GHz}$ due to optical depth effects.

White et al. (1989) investigated simple non-thermal models for the quiescent radio emission from dMe flare stars, using an analytic representation of the magnetic field and spatial inhomogeneities in the nonthermal electron density distribution. The magnetic field is assumed to be dipolar $\left(B(r) \propto r^{-n}\right.$, where $\left.n=3\right)$. The distribution of electrons with energy is parameterized as a power-law with index $\delta$, and is assumed to vary radially with distance as $N=N^{\prime}\left(E_{0}\right)\left(r / r_{0}\right)^{-m}$, where $N^{\prime}\left(E_{0}\right)$ is the electron density distribution. Combining these assumptions with the analytic expressions for absorption and emission coefficients of Dulk \& 
Marsh (1982), expressions for the optically thick and thin radiative fluxes can be obtained ${ }^{2}$. White et al. (1989) considered a global dipole, which they termed a "bare" dipole, and a "buried dipole", in which the depth below the surface at which the dipole is buried is much less than a stellar radius. White et al. (1989) compared their analytic expressions with more accurate numerical calculations and conclude that both are within a factor of two of the numerical calculations. We use the analytic expressions to obtain a general description of coronal conditions which could be applicable to the case of TVLM513.

In our case, we calculate the expected fluxes at the distance of TVLM513 (10.5 pc), specifying the frequency $(1.4,5$, and $8 \mathrm{GHz})$, constraining $\delta$ to be 3 , and assuming only dipolar field configurations. For a harder electron energy spectrum $(\delta=2)$ the total integrated energy in fast electrons does not converge. This leaves four free parameters: the power-law index describing the radial dependence of the number density $(m)$, the base magnetic field strength $\left(B_{0}\right)$, the total surface number density $\left(N_{0}\right)$, and the scale length of the dipole $\left(=x \times R_{\star}\right.$, where $x$ is $\left.\leq 1\right)$. The $m=0$ case corresponds to a nonthermal electron distribution independent of radius, appropriate for an isotropic pitch angle distribution. The other extreme, $m=n=3$, corresponds to the radial dependence of the electrons being the same as that of the dipolar magnetic field; this is the situation that would be obtained by conserving both particle and magnetic flux in the case of open field lines. We require that the turnover frequency be $<5 \mathrm{GHz}$, based on the observational constraints. Figure 2 shows the range of parameter space in base magnetic field strength and total nonthermal number density, for different combinations of $m$ and bare/buried dipoles which predict between 0.5 and 2 times the detected flux at $3.6 \mathrm{~cm}$. For $\delta=3$, the predicted flux varies as $S \propto N B^{2.5}$. The turnover frequency likewise depends more strongly on the base magnetic field strength than the total number density of accelerated electrons. This places a constraint on the base magnetic field strength to be less than $~ 500 \mathrm{G}$ to satisfy the observed fluxes and spectral distribution. Requiring the dipole to be buried at depths less than the stellar radius increases the number density of accelerated electrons needed to produce the same observed flux.

The radio observations indicate little net polarization, but do not provide a stringent constraint on the global magnetic geometry: the upper limits on polarization could be consistent with small regions of magnetic field permeating the surface, or a large dipolar field with high inclination so that the net circular polarization signature is small. Mutel et al. (1987) noted a relationship for RS CVn binary systems (known to have large-scale magnetic fields) in which the measured circular polarizations decrease with increasing inclination. The high rotational velocity might also suggest a nearly edge-on viewing geometry to see the maximum

\footnotetext{
${ }^{2}$ There is an error in White et al. (1989) equation 7: a plus sign in the third line of the formula should be a multiplication symbol.
} 
rotational broadening.

\section{Conclusions}

There are various lines of evidence which suggest that similar activity is being displayed on late $\mathrm{M}$ dwarfs as is seen in the earlier $\mathrm{M}$ dwarfs: radio flares; transition region and X-ray emission (including flaring at these wavelengths); and sporadic detections of $\mathrm{H} \alpha$ emission and flaring. To this list we can add quiescent radio emission with typical spectral indices of earlier M dwarfs and little to no circular polarization. Longer term observations of ultracool dwarfs are needed to understand fully the nature of the radio emission and how commonly it occurs in these objects. A more comprehensive view of activity diagnostics in ultracool dwarfs is also needed to make sense of the broad changes seen in $\mathrm{H} \alpha$ emission trends, and to relate them to the physical changes taking place in these ultracool dwarf atmospheres.

This paper represents the results of VLA project AO180. RAO acknowledges support from a Jansky Postdoctoral Fellowship from the National Radio Astronomy Observatory. SLH acknowledges support from NSF grant AST02-11559. 


\section{REFERENCES}

Berger, E. 2002, ApJ, 572, 503

Berger, E., Ball, S., Becker, K. M., Clarke, M., Frail, D. A., Fukuda, T. A., Hoffman, I. M., Mellon, R., Momjian, E., Murphy, N. W., Teng, S. H., Woodruff, T., Zauderer, B. A., \& Zavala, R. T. 2001, Nature, 410, 338

Close, L. M., Siegler, N., Freed, M., \& Biller, B. 2003, ApJ, 587, 407

Dulk, G. A. 1985, ARA\&A, 23, 169

Dulk, G. A. \& Marsh, K. A. 1982, ApJ, 259, 350

Fleming, T. A., Giampapa, M. S., \& Garza, D. 2003, ApJ, 594, 982

Güdel, M. \& Benz, A. O. 1996, in Astronomical Society of the Pacific Conference Series, $303-+$

Hawley, S. L. \& Johns-Krull, C. M. 2003, ApJ, 588, L109

Leggett, S. K., Allard, F., Geballe, T. R., Hauschildt, P. H., \& Schweitzer, A. 2001, ApJ, 548,908

Liebert, J., Kirkpatrick, J. D., Cruz, K. L., Reid, I. N., Burgasser, A., Tinney, C. G., \& Gizis, J. E. 2003, AJ, 125, 343

Mohanty, S. \& Basri, G. 2003, ApJ, 583, 451

Mohanty, S., Basri, G., Shu, F., Allard, F., \& Chabrier, G. 2002, ApJ, 571, 469

Mutel, R. L., Morris, D. H., Doiron, D. J., \& Lestrade, J. F. 1987, AJ, 93, 1220

Pont, F., Melo, C. H. F., Bouchy, F., Udry, S., Queloz, D., Mayor, M., \& Santos, N. C. 2005, astro-ph/0501611

Reid, I. N., Kirkpatrick, J. D., Liebert, J., Gizis, J. E., Dahn, C. C., \& Monet, D. G. 2002, AJ, 124, 519

Rutledge, R. E., Basri, G., Martín, E. L., \& Bildsten, L. 2000, ApJ, 538, L141

Tinney, C. G., Mould, J. R., \& Reid, I. N. 1993, AJ, 105, 1045

West, A. A., Hawley, S. L., Walkowicz, L. M., Covey, K. R., Silvestri, N. M., Raymond, S. N., Harris, H. C., Munn, J. A., McGehee, P. M., Ivezić, Ž., \& Brinkmann, J. 2004, AJ, 128,426 
White, S. M., Kundu, M. R., \& Jackson, P. D. 1989, A\&A, 225, 112 
Table 1. Radio Parameters

\begin{tabular}{llcccccc}
\hline \hline $\begin{array}{c}\lambda \\
(\mathrm{cm})\end{array}$ & $\begin{array}{c}\text { Freq. } \\
(\mathrm{GHz})\end{array}$ & $\begin{array}{c}\mathrm{T}_{\text {int }} \\
(\mathrm{s})\end{array}$ & \multicolumn{1}{c}{$\begin{array}{c}\text { Beam } \\
\text { "x" }\end{array}$} & $\begin{array}{c}\text { I Flux } \\
(\mu \mathrm{Jy})\end{array}$ & $\begin{array}{c}\text { V Flux } \\
(\mu \mathrm{Jy})\end{array}$ & $\begin{array}{c}\pi_{c} \\
\%\end{array}$ & $\begin{array}{c}\mathrm{L}_{\text {rad }} \\
\left(\mathrm{erg} \mathrm{s}^{-1} \mathrm{~Hz}^{-1}\right)\end{array}$ \\
\hline 3.6 & 8.4 & 10510 & $2.8 \times 2.2, \mathrm{PA}=71$ & $228 \pm 11$ & $<33$ & $<15$ & $3.0 \times 10^{13}$ \\
6 & 4.8 & 10520 & $4.4 \times 3.2, \mathrm{PA}=80$ & $284 \pm 13$ & $<42$ & $<15$ & $3.8 \times 10^{13}$ \\
20 & 1.4 & 10580 & $13.3 \times 10.1, \mathrm{PA}=24$ & $260 \pm 46$ & $<68$ & $<26$ & $3.5 \times 10^{13}$ \\
\hline
\end{tabular}

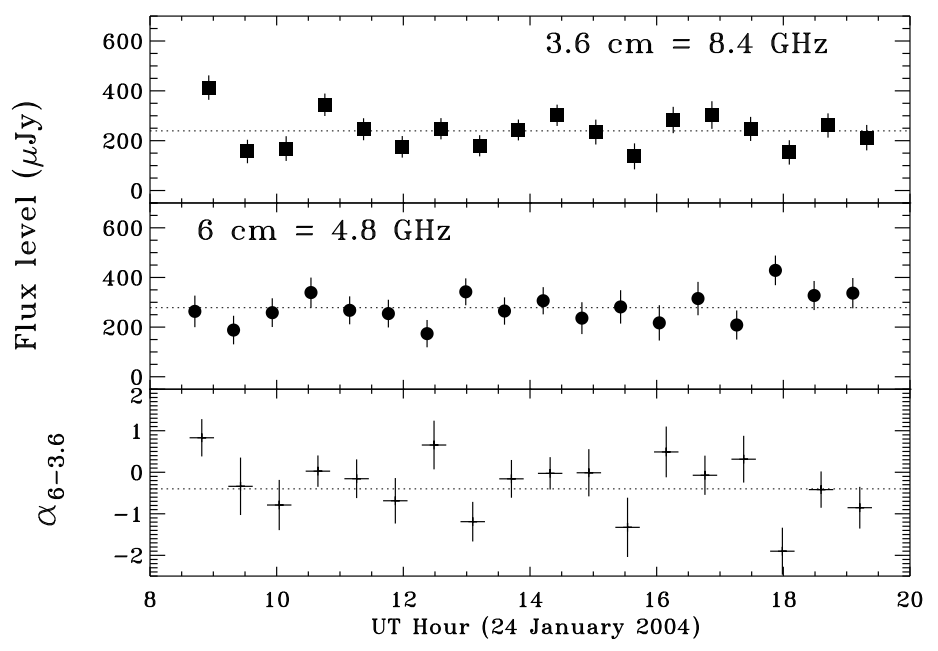

Fig. 1.- Variation of 3.6 and $6 \mathrm{~cm}$ fluxes during the $\sim 10$ hour observation. Each time bin corresponds to an average over each scan, approximately 10 minutes in length. The dotted lines indicate the average flux density during the observation. Bottom panel shows variation of 3.6-6 cm spectral index during the observation; dotted line indicates spectral index determined from entire observation. 


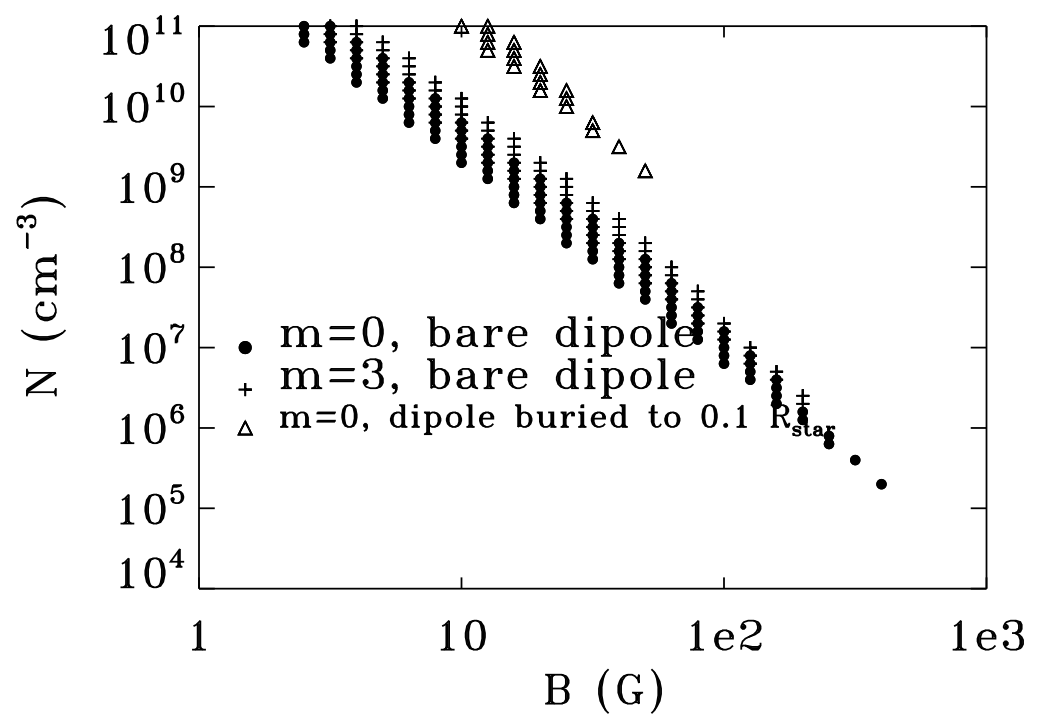

Fig. 2.- Constraints on dipole magnetic field strength and nonthermal electron density in the corona of TVLM513, under different assumptions. Here, $m$ is the index of the power-law radial dependence of the nonthermal electron density, and we consider dipoles whose scale equals the stellar radius ("bare dipole") and those with scale lengths less than the stellar radius ("buried dipole"). Allowed models are those which predict between 0.5 and 2 times the detected flux at $3.6 \mathrm{~cm}$, and are optically thin at frequencies higher than $5 \mathrm{GHz}$. 